\title{
CONSISTENT PARTITIONS OF POLYTOPES AND POLYNOMIAL MEASURES
}

\author{
A. KhovanskiI
}

Let $\Delta_{1}, \ldots, \Delta_{n}$ be $n$ polytopes in $\mathbb{R}^{n}$. There are many ways to decompose the polytopes $\Delta_{i}$ into smaller polytopes $\Gamma_{i}^{j}$ and then choose certain "consistent"

collections that contain a single polytope $\Gamma_{i}^{j_{i}}$ from each polytope $\Delta_{i}$ in such a way that the following formula holds:

$$
V\left(\Delta_{1}, \ldots, \Delta_{n}\right)=\sum V\left(\Gamma_{1}^{j_{1}}, \ldots, \Gamma_{n}^{j_{n}}\right),
$$

where $V$ is the mixed volume, and the summation is taken over all consistent collections. The theory of Newton polytopes provides a lot of examples of this kind (see $\S 2$ ). The present paper arose from attempts to clarify whether there exists a similar effect for other finitely additive measures that differ from the volume measure (for instance, for the number of integral points of a polytope).

In the paper we introduce the notion of consistent partition for several polytopes (whose number can differ from the dimension of the underlying space). The main result of the present paper is the generalization of relation $(*)$ to any consistent partition and for any finitely additive measure (see $\S 3$ ).

Consider a measure on the integral polytopes that is polynomial with respect to the shifts of the polytopes by the vectors of the integral lattice. As is known, such a measure is a polynomial on the semigroup of integral polytopes with respect to the Minkowski addition. For the measures invariant with respect to shifts by the vectors of the integral lattice, this result is due to McMullen [6]. It was extended to the case of polynomial measures with respect to the shifts in [5], where the integral over the Euler characteristic was used. Here we show that the result is the direct consequence of the generalized relation $(*)$.

\section{$\S 1$. Consistent Regular Partitions}

In the paper, by a polytope we mean a compact convex polytope, that is, the convex hull of a finite sets of points. By a partition of a polytope $\Delta$ we mean a finite set of polytopes $\mathcal{R}(\Delta)$ such that

1) the union of the polytopes from $\mathcal{R}(\Delta)$ is the polytope $\Delta$,

2 ) the set $\mathcal{R}(\Delta)$ contains all faces of any polytope from $\mathcal{R}(\Delta)$, and

$3)$ the intersection of any two polytopes from $\mathcal{R}(\Delta)$ is either empty or a face of these polytopes.

This research was completed during a visit to the Ecole Normale Supérieure and partially supported by the Russian Foundation for Basic Research under Grant No. 95-011-8701 and by the Canadian Grant OGP0156833. The author thanks his French colleagues for their warm hospitality. 
The simplest example of a partition is the tautological partition of a polytope that contains the polytope and all its faces.

Among the partitions of a polytope, regular partitions are selected [3]. First, this class of partitions is closely related to the theory of Newton polytopes (see [7]), and, secondly, it is flexible and convenient to work with. Let us pass to the definition of this class of partitions.

Let $\Delta$ be a polytope in a linear space $L_{1}$. We say that a polytope $\widetilde{\Delta}$ that belongs to the product of the space $L_{1}$ and the real line $\mathbb{R}^{1}$ lies over the polytope $\Delta$ if $\Delta$ is the projection of $\widetilde{\Delta}$. We assume that an orientation is chosen in $\mathbb{R}^{1}$. Let $e_{1}$ be a basis vector in $\mathbb{R}^{1}$. We say that a point $x \in \widetilde{\Delta}$ is an upper point of $\widetilde{\Delta}$ if the ray $x+\lambda e_{1}$, where $\lambda \geqslant 0$, intersects $\widetilde{\Delta}$ at the point $x$ only. A face of the polytope $\widetilde{\Delta}$ is called an upper face if each of its points is an upper point of this polytope.

Obviously, a face $\Gamma$ of the polytope $\widetilde{\Delta}$ is an upper face if and only if there exists a covector $\xi \in\left(L_{1} \times \mathbb{R}^{1}\right)^{*}$ for which $\left\langle\xi, e_{1}\right\rangle>0$ and such that the maximum on $\widetilde{\Delta}$ of the inner product with the covector $\xi$ is attained on the face $\Gamma$.

By a partition of a polytope $\Delta$ related to a polytope $\widetilde{\Delta}$ that lies over $\Delta$ we mean the partition $\mathcal{R}(\Delta)$ that consists of the projections of the upper faces of $\widetilde{\Delta}$. A partition of $\Delta$ related to some polytope $\widetilde{\Delta}$ is said to be regular.

Note that a regular partition of a polytope $\Delta$ depends not on $\widetilde{\Delta}$ itself but only on the upper points of $\widetilde{\Delta}$. The set of upper points of $\widetilde{\Delta}$ can be regarded as the graph of a convex piecewise linear function on the polytope $\Delta$. Conversely, to each of the convex piecewise linear functions on the polytope $\Delta$, a regular partition is assigned, namely, for the corresponding polytope $\widetilde{\Delta}$ we must take the convex hull of the graph of this function.

Let $\mathcal{R}\left(\Delta_{1}\right), \ldots, \mathcal{R}\left(\Delta_{k}\right)$ be a set of regular partitions of the polytopes $\Delta_{1}, \ldots, \Delta_{k}$. Let us choose polytopes $\widetilde{\Delta}_{1}, \ldots, \widetilde{\Delta}_{k}$ that lie over $\Delta_{1}, \ldots, \Delta_{k}$, respectively, and generate the partitions $\mathcal{R}\left(\Delta_{1}\right), \ldots, \mathcal{R}\left(\Delta_{k}\right)$. The choice of the polytopes $\widetilde{\Delta}_{i}$ determines the consistency of the regular partitions.

We say that the polytopes $\Gamma_{1}, \ldots, \Gamma_{k}$ from the partitions $\mathcal{R}\left(\Delta_{1}\right), \ldots, \mathcal{R}\left(\Delta_{k}\right)$ are regularly consistent (for the polytopes $\widetilde{\Delta}_{i}$ ) if the Minkowski sum $\widetilde{\Gamma}_{1}+\cdots+\widetilde{\Gamma}_{k}$ of the upper faces $\widetilde{\Gamma}_{i}$ (of the polytopes $\widetilde{\Delta}_{i}$ ) that lie over $\Gamma_{i}$ is an upper face $\widetilde{\Gamma}$ of the polytope $\widetilde{\Delta}=\widetilde{\Delta}_{1}+\cdots+\widetilde{\Delta}_{n}$. Thus, to each of the upper faces $\widetilde{\Gamma}$ of $\widetilde{\Delta}$ we assign a unique set of consistent polytopes $\Gamma_{1}, \ldots, \Gamma_{k}$.

An equivalent definition can be given as follows. Polytopes $\Gamma_{1}, \ldots, \Gamma_{k}$ from partitions $\mathcal{R}\left(\Delta_{1}\right), \ldots, \mathcal{R}\left(\Delta_{k}\right)$ are said to be regularly consistent (for the polytopes $\left.\widetilde{\Delta}_{i}\right)$ if there exists a linear function $l: L_{1} \times \mathbb{R}^{1} \rightarrow \mathbb{R}^{1}$ whose maximum on the $i$ th polytope $\widetilde{\Delta}_{i}$ is attained exactly on the upper face $\widetilde{\Gamma}_{i}$ of the polytope $\widetilde{\Delta}_{i}$ that lies over $\Gamma$.

We say that the polytopes $\Delta_{1}, \ldots, \Delta_{k}$ are affinely independent if the minimal affine subspaces containing these polytopes are independent. In other words, $\Delta_{1}, \ldots, \Delta_{k}$ are affinely independent if the dimension of their Minkowski sum is equal to the sum of their dimensions:

$$
\operatorname{dim}\left(\Delta_{1}+\cdots+\Delta_{k}\right)=\operatorname{dim} \Delta_{1}+\cdots+\operatorname{dim} \Delta_{k}
$$


Theorem. Any collection $\mathcal{R}\left(\Delta_{1}\right), \ldots, \mathcal{R}\left(\Delta_{k}\right)$ of regular partitions can be made regularly consistent in such a way that any consistent collection of polytopes will be affinely independent.

Proof. Let a regular partition of the polytope $\Delta$ be assigned to a convex piecewise linear function $f: \Delta \rightarrow \mathbb{R}$. If we add a linear function to the function $f$, then the corresponding regular partition remains unchanged. Let the partition $\mathcal{R}\left(\Delta_{i}\right)$ be assigned to a function $f_{i}: \Delta_{i} \rightarrow \mathbb{R}$. We can readily see that by adding sufficiently general linear functions $L_{i}$ to the functions $f_{i}$, we can make any consistent collection of polytopes affinely independent.

Corollary. The tautological partitions of several polytopes in $\mathbb{R}^{n}$ can be made regularly consistent in such a way that any consistent collection will contain the affinely independent faces of these polytopes.

Proof. Indeed, the tautological partition is clearly regular because it is assigned to any linear function on the polytope.

\section{§2. Newton polytopes AND MiXed VOlumes}

In this section we discuss the relation between algebraic geometry and the problems that are studied in the paper. This discussion here are not used in the sequel and this section can be omitted at the first reading.

Consider the system of $n$ equations

$$
P_{1}=\cdots=P_{n}=0
$$

in $\left(\mathbb{C}^{*}\right)^{n}$, where $P_{1}, \ldots, P_{n}$ are Laurent polynomials that are sufficiently general for their Newton polytopes $\Delta_{1}, \ldots, \Delta_{n}$. The connection between measure theory and algebraic geometry is provided by the Bernshtein theorem [1] which states that the number of solutions of system (1) is $n ! V\left(\Delta_{1}, \ldots, \Delta_{n}\right)$.

Denote by $a_{i j}$ the coefficients of the Laurent polynomial $P_{i}, P_{i}=\sum a_{i j} x^{j}$, $j \in \mathbb{Z}^{n}$. Consider arbitrary Laurent polynomials $a_{i j}(\tau)$ in one variable $\tau$ such that $a_{i j}(1)=a_{i j}$.

We can regard the system of equations

$$
\widetilde{P}_{1}(x, \tau)=\cdots=\widetilde{P}_{n}(x, \tau)=0,
$$

in which we set $\widetilde{P}_{i}(x, \tau)=\sum a_{i j}(\tau) x^{j}$, as a system of equations with unknown point $x \in\left(\mathbb{C}^{*}\right)^{n}$ and with parameter $\tau$. For $\tau=1$, system (2) coincides with system (1) and has the same roots. The theory of Newton polytopes makes it possible to describe the behavior of the roots of system (2) as $\tau \rightarrow \infty$, at least in the case in which the Laurent polynomials $\widetilde{P}_{1}, \ldots, \widetilde{P}_{n}$ are sufficiently general for their Newton polytopes $\widetilde{\Delta}_{1}, \ldots, \widetilde{\Delta}_{n}$.

Namely, consider an arbitrary upper face $\widetilde{\Gamma}$ of the polytope $\widetilde{\Delta}=\widetilde{\Delta}_{1}+\cdots+\widetilde{\Delta}_{n}$. This face is the sum of certain upper faces $\widetilde{\Gamma}_{1}, \ldots, \widetilde{\Gamma}_{n}$ of $\widetilde{\Delta}_{1}, \ldots, \widetilde{\Delta}_{n}$. To any upper face $\widetilde{\Gamma}$ we assign the system of equations

$$
\widetilde{P}_{1}^{\widetilde{\Gamma}_{1}}(x, \tau)=\cdots=\widetilde{P}_{n}^{\widetilde{\Gamma}_{n}}(x, \tau)=0,
$$

where $\widetilde{P}_{i} \widetilde{\Gamma}_{i}$ is the truncation of the Laurent polynomial $\widetilde{P}_{i}$ with respect to the face $\widetilde{\Gamma}_{i}[4]$. We obtain as many systems as there are upper faces of the polytope $\widetilde{\Delta}$.

Let $\tau:\left(\mathbb{R}^{1}, 0\right) \rightarrow(\widetilde{\mathbb{C}}, \infty)$ be a germ of a real curve on the Riemann sphere such that $\tau(0)=\infty$. Consider the roots of system (1) for $\tau=\tau(t)$ as $t \rightarrow 0$ and $\tau(t) \rightarrow \infty$. 
Theorem (on the asymptotic solution of system (1), cf. [1, 4]). If the Laurent polynomials $\widetilde{P}_{1}, \ldots, \widetilde{P}_{n}$ are $\Delta$-nondegenerate with respect to their Newton polytopes, then, on a germ $\tau(t)$ of a curve, there exists a one-to-one correspondence between the set $\{x(\tau(t))\}$ of all roots of system (1) and the union $\{\widetilde{x}(\tau(t))\}$ of the roots of systems $(3 \widetilde{\Gamma})$ over all upper faces $\widetilde{\Gamma}$ of the polytope $\widetilde{\Delta}$. Here the ratio $x \widetilde{x}^{-1}$, in the group $\left(\mathbb{C}^{*}\right)^{n}$, of the mutually corresponding roots $x$ and $\widetilde{x}$ tends to the identity element of the group $\left(\mathbb{C}^{*}\right)^{n}$ as $t \rightarrow 0$ and $\tau(t) \rightarrow \infty$.

In particular, it follows from this theorem that the number of solutions of system (1) is equal to the sum of the numbers of solutions of systems $\left(3_{\widetilde{\Gamma}}\right)$ for a chosen value $\tau\left(t_{0}\right)$ of the parameter $\tau$, where $t_{0}$ is a small number. Denote by $\Gamma_{i} \subset \Delta_{i}$ the projection of the face $\widetilde{\Gamma}_{i}$ of $\widetilde{\Delta}_{i}$. For $\tau=\tau\left(t_{0}\right)$, the Newton polytopes of system $\left(3_{\widetilde{\Gamma}}\right)$ are the projections $\Gamma_{1}, \ldots, \Gamma_{n}$ of the faces $\widetilde{\Gamma}_{1}, \ldots, \widetilde{\Gamma}_{n}$. By applying the Bernshtein theorem to these systems, we obtain the following assertion.

Corollary. Under the above assumptions, we have the relation

$$
n ! V\left(\Delta_{1}, \ldots, \Delta_{n}\right)=\sum n ! V\left(\Gamma_{1}, \ldots, \Gamma_{n}\right),
$$

where the sum is taken over all collections $\Gamma_{1}, \ldots, \Gamma_{n}$ of corresponding regular partitions of the polytopes $\Delta_{1}, \ldots, \Delta_{n}$ that are consistent by means of the polytopes $\widetilde{\Delta}_{1}, \ldots, \widetilde{\Delta}_{n}$.

Remark 1 . If for some face $\widetilde{\Gamma}$ of the polytope $\widetilde{\Delta}$, where $\widetilde{\Gamma}=\widetilde{\Gamma}_{1}+\cdots+\widetilde{\Gamma}_{n}, \widetilde{\Gamma}_{i} \in \widetilde{\Delta}_{i}$, a face $\widetilde{\Gamma}_{j}$ is a vertex, then the mixed volume $V\left(\Gamma_{1}, \ldots, \Gamma_{n}\right)$ of the corresponding polytopes is zero. To this face $\widetilde{\Gamma}$, an inconsistent system $\left(3_{\widetilde{\Gamma}}\right)$ is assigned.

Remark 2 . The Laurent polynomials $a_{i j}(\tau)$ can be chosen so that all systems $\left(3_{\widetilde{\Gamma}}\right)$ can be solved explicitly. To this end, it suffices to take any Laurent polynomial $\widetilde{P}_{i}^{\widetilde{\Gamma}_{i}}$ (for each of the faces $\widetilde{\Gamma} \subset \widetilde{\Delta}$ such that the corresponding system $\left(3_{\widetilde{\Gamma}}\right)$ is consistent) as the linear combination of two monomials. In this case, the projection of the face $\widetilde{\Gamma}$ is a parallelepiped, and the number of solutions of system $\left(3_{\widetilde{\Gamma}}\right)$ is equal to the volume of this parallelepiped multiplied by $n$ !. Applying this argument, we can not only prove the Bernshtein theorem, but also obtain new formulas for the mixed volume (which generalize formulas from [3]). I will return to this subject elsewhere.

\section{§3. Consistent partitions of several polytopes}

Our nearest goal is the general definition of the consistency of (not necessarily regular) partitions of $k$ polytopes. Two approaches to the definition are possible. The first is based on the "multidimensional" point of view and uses the join of $k$ polytopes. The other approach uses the point of view of the underlying space of the $k$ polytopes. We begin with the multidimensional viewpoint.

By the join $\Omega$ of polytopes $\Delta_{1}, \ldots, \Delta_{k}$ which lie in a linear space $L_{1}$, over a simplex of dimension $k-1$ with vertices $A_{1}, \ldots, A_{k}$, which lies in a space $L_{2}$, we mean the convex hull of the polytopes $\left(\Delta_{i}, A_{i}\right) \subset L_{1} \times L_{2}$.

In what follows we give a description of the faces of the join of polytopes $\Delta_{1}, \ldots, \Delta_{k}$. We need the following definition. A collection of faces $\Gamma_{1}, \ldots, \Gamma_{k}$ of polytopes $\Delta_{1}, \ldots, \Delta_{k} \subset L$ is said to be consistent if there exists a covector $\xi \in L^{*}$ such that the maximum on $\Delta_{i}$ of the inner product with the covector $\xi$ is attained precisely on the face $\Gamma_{i}$.

The following assertion can readily be verified. 
Proposition 1. To any subset $\Delta_{i_{1}}, \ldots, \Delta_{i_{l}}$ of the set of polytopes $\Delta_{1}, \ldots, \Delta_{k}$ and to each of consistent collections of faces $\Gamma_{i_{1}} \subset \Delta_{i_{1}}, \ldots, \Gamma_{i_{l}} \subset \Delta_{i_{l}}$, there corresponds a face of the join $\Omega$ of the polytopes $\Delta_{1}, \ldots, \Delta_{l}$. Namely, this face is the join of the faces $\Gamma_{i_{1}}, \ldots, \Gamma_{i_{l}}$ over the simplex with the vertices $A_{i_{1}}, \ldots, A_{i_{l}}$. Conversely, any face of the join $\Omega$ has the above form.

A partition $\mathcal{R}(\Omega)$ of the join $\Omega$ of the polytopes $\Delta_{1}, \ldots, \Delta_{k}$ over a simplex in a space $L_{2}$ with vertices $A_{1}, \ldots, A_{k}$ is said to be correct if the projection of any vertex of this partition to the space $L_{2}$ is one of the vertices $A_{1}, \ldots, A_{k}$. A correct partition $\mathcal{R}(\Omega)$ of the join defines certain partitions $\mathcal{R}\left(\Delta_{1}\right), \ldots, \mathcal{R}\left(\Delta_{k}\right)$ of the polytopes $\Delta_{1}, \ldots, \Delta_{k}$. To construct the partition $\mathcal{R}\left(\Delta_{i}\right)$, we must identify the polytope $\Delta_{i}$ with the face $\left(\Delta_{i}, A_{i}\right)$ of the join $\Omega$ and consider the partition of this face induced by the partition $\mathcal{R}(\Omega)$. In this case we also say that a correct partition $\mathcal{R}(\Omega)$ of the join defines a consistency of the partitions $\mathcal{R}\left(\Delta_{1}\right), \ldots, \mathcal{R}\left(\Delta_{k}\right)$ of the polytopes $\Delta_{1}, \ldots, \Delta_{k}$.

Denote by $\mathcal{R}(\Omega, k)$ the subset of the polytopes of a correct partition $\mathcal{R}(\Omega)$ whose vertices are projected into the set $A_{1}, \ldots, A_{k}$. It is clear that any polytope $\Gamma \in \mathcal{R}(\Omega, k)$ is the join of the corresponding polytopes $\Gamma_{1}, \ldots, \Gamma_{k}$ from the partitions $\mathcal{R}\left(\Delta_{1}\right), \ldots, \mathcal{R}\left(\Delta_{k}\right)$. The set $\mathcal{R}(\Omega, k)$ completely defines the partition $\mathcal{R}(\Omega)$ : namely, $\mathcal{R}(\Omega)$ contains only the polytopes from $\mathcal{R}(\Omega, k)$ and their faces.

The set $\mathcal{R}(\Omega, k)$ can be defined by introducing the following subset $S$ in the Cartesian product $\mathcal{R}\left(\Delta_{1}\right) \times \cdots \times \mathcal{R}\left(\Delta_{k}\right)$ : a collection of polytopes $\left(\Gamma_{1}(s), \ldots, \Gamma_{k}(s)\right)$, where $\Gamma_{i} \in \mathcal{R}\left(\Delta_{i}\right)$, is a point $s$ of the set $S$ if and only if the set $\mathcal{R}(\Omega, k)$ contains the join of the polytopes $\Gamma_{1}, \ldots, \Gamma_{k}$ over the simplex with vertices $A_{1}, \ldots, A_{k}$.

The set $S \subset \mathcal{R}\left(\Delta_{1}\right) \times \cdots \times \mathcal{R}\left(\Delta_{k}\right)$ has the following properties:

1) for any $k$-tuple of positive reals $\left(\lambda_{1}, \ldots, \lambda_{k}\right)=\lambda$, the set of polytopes $\Gamma(s, \lambda)=\lambda_{1} \Gamma_{1}(s)+\cdots+\lambda_{k} \Gamma_{k}(s)$ is a partition, say, $\mathcal{R}(\Delta(\lambda))$, of the polytope $\Delta(\lambda)=\lambda_{1} \Delta_{1}+\cdots+\lambda_{k} \Delta_{k} ;$ moreover, each polytope $\Gamma \in \mathcal{R}(\Delta(\lambda))$ coincides with a polytope of the form $\Gamma(s, \lambda)$ for exactly one $s \in S$;

2) for any covector $\xi$ and any element $s_{1} \in S$, there exists an element $s_{2} \in S$ such that $\Gamma_{i}\left(s_{2}\right)=\Gamma_{i}^{\xi}\left(s_{1}\right)$.

Conversely, we can define the consistency of the partitions $\mathcal{R}\left(\Delta_{1}\right), \ldots, \mathcal{R}\left(\Delta_{k}\right)$ by choosing a subset $S$ of the Cartesian product $\mathcal{R}\left(\Delta_{1}\right) \times \cdots \times \mathcal{R}\left(\Delta_{k}\right)$ with properties 1) and 2). This gives the second ("low-dimensional") description of the consistency of partitions of polytopes.

Proposition 2. A regular consistency of regular partitions of several polytopes is a consistency of these polytopes.

Proof. Let a regular consistency of regular partitions of polytopes $\Delta_{1}, \ldots, \Delta_{k}$ is related to convex piecewise linear functions $f_{1}, \ldots, f_{k}$. Consider a convex piecewise linear function $F$ on the join $\Omega$ of these polytopes, where $F$ is defined as follows: the convex hull of the graph of the function $F$ coincides with the convex hull of the graphs of the functions $f_{i}$ that are given on the faces $\left(\Delta_{i}, A_{i}\right)$ of the join $\Omega$. We can readily verify that the regular partition of the join related to the function $F$ defines a consistency of the regular partitions of polytopes $\Delta_{1}, \ldots, \Delta_{k}$ that coincides with their regular consistency related to the functions $f_{1}, \ldots, f_{k}$.

\section{$\S 4$. Partitions and the Euler characteristic}

Now we need the following Propositions 3 and 4, which are related to the Euler 
characteristics and partitions of polytopes.

Denote by $\chi_{\Delta}$ the characteristic function of an arbitrary polytope $\Delta$.

Proposition 3. For each polytope $\Delta$ and any of its partitions $\mathcal{R}(\Delta)$, the following relation holds:

$$
\chi_{\Delta}=\sum_{\Gamma \in \mathcal{R}(\Delta), \Gamma \not \subset \partial \Delta}(-1)^{\operatorname{codim} \Gamma} \chi_{\Gamma} .
$$

(The boundary $\partial \Delta$ of a polytope $\Delta$ is always taken in the topology of the minimal affine subspace that contains the polytope $\Delta$.)

Proof. Denote by $B(x, \varepsilon)$ the ball of radius $\varepsilon$ with center at the point $x$. For each point $x \in \Delta$, the intersection $B(x, \varepsilon) \cap \Delta$ is a closed convex set whose dimension is equal to that of the polytope $\Delta$. The Euler characteristic of the relative homology groups of the set $B(x, \varepsilon) \cap \Delta$ with respect to its boundary $\partial(B(x, \varepsilon) \cap \Delta)$ is equal to $(-1)^{\operatorname{dim} \Delta}$. For a sufficiently small $\varepsilon$, the Euler characteristic of the relative homology can be calculated by means of the cells $B(x, \varepsilon) \cap \Gamma$, where $\Gamma$ is an arbitrary polytope from the partition $\mathcal{R}(\Delta)$ that contains the point $x$ and does not belong entirely to the boundary of the set $B(x, \varepsilon) \cap \Delta$. This yields

$$
(-1)^{\operatorname{dim} \Delta} \chi_{\Delta}(x)=\sum_{x \in \Gamma, \Gamma \in \mathcal{R}(\Delta), \Gamma \not \subset \partial \Delta}(-1)^{\operatorname{dim} \Gamma} \chi_{\Gamma}(x),
$$

or $\chi_{\Delta}(x)=\sum(-1)^{\operatorname{codim} \Gamma} \chi_{\Gamma}(x)$, and the proof is complete.

Corollary 1. For any finitely additive measure $\mu$ such that all polytopes from a partition $\mathcal{R}(\Delta)$ are measurable, the following relation holds:

$$
\mu(\Delta)=\sum_{\Gamma \in \mathcal{R}(\Delta), \Gamma \not \subset \partial \Delta}(-1)^{\operatorname{codim} \Gamma} \mu(\Gamma) .
$$

Proposition 4. For each partition $\mathcal{R}(\Delta)$ of a polytope $\Delta$, the following identity holds:

$$
1=\sum_{\Gamma \in \mathcal{R}(\Delta), \Gamma \not \subset \partial \Delta}(-1)^{\operatorname{codim} \Gamma} .
$$

Proof. The identity follows from the general topological fact: the Euler characteristic of the pair $(\Delta, \partial \Delta)$ is equal to $(-1)^{\operatorname{dim} \Delta}$.

Remark. Certainly, Proposition 4 is related to Corollary 1. The point is that there exists a finitely additive measure on the Boolean algebra generated by the convex polytopes whose value on any polytope is equal to its Euler characteristic, i.e., is equal to one $[8,5]$. We shall formally use this fact in the proof of the main theorem (see $\S 5$ ). However, in fact we shall apply Proposition 4 for a correct partition of a join only.

Let us apply these facts to the partition $\mathcal{R}(\Omega)$ of the join of $k$ polytopes (see $\S 3$ ). The set $\mathcal{R}(\Omega, k)$ has a subset $\mathcal{R}_{0}(\Omega, k)$ that consists of the polytopes $\Gamma$ for which the corresponding polytopes $\Gamma_{1}, \ldots, \Gamma_{k}$ do not belong to the consistent faces of the polytopes $\Delta_{1}, \ldots, \Delta_{k}$. In other words, this subset consists of the polytopes $\Gamma$ for which there exists no nonzero covector $\xi$ such that $\Gamma_{i} \subset \Delta_{i}^{\xi}$.

The description of the faces of the join (Proposition 1), Proposition 4, and Corollary 1 of the present section imply the following assertion. 
Corollary 2. 1. For any finitely additive measure $g$ such that all polytopes $\Gamma$ from the partition $\mathcal{R}(\Delta)$ are measurable, the following relation holds:

$$
g(\Omega)=\sum_{\Gamma \in \mathcal{R}_{0}(\Omega, k)}(-1)^{\operatorname{codim} \Gamma} g(\Gamma) .
$$

2. We have

$$
1=\sum_{\Gamma \subset \mathcal{R}_{0}(\Omega, k)}(-1)^{\operatorname{codim} \Gamma} .
$$

Our main theorem (see $\S 5$ ) is proved by applying Corollary 2 to a special measure $g$ on $L_{1} \times L_{2}$ constructed by a given measure $\mu$ on the space $L_{1}$.

\section{§5. MAIn THEOREM}

Let $\mathcal{R}(\Omega)$ be an arbitrary consistent partition of polytopes $\Delta_{1}, \ldots, \Delta_{k}$ in the space $L_{1}$, and let all polytopes in each partition $\mathcal{R}\left(\Delta_{i}\right)$ be measurable with respect to a finitely additive measure $\mu$. Let the Boolean algebra $\mathcal{B}_{\mu}$ of the sets that are measurable with respect to $\mu$ have the following property: if it contains convex polytopes $\Delta_{1}$ and $\Delta_{2}$, then it contains their Minkowski sum $\Delta_{1}+\Delta_{2}$ as well. Under these assumptions we have the following assertion.

Main Theorem. For any measurable consistent partition $\mathcal{R}(\Omega)$ of the polytopes $\Delta_{1}, \ldots, \Delta_{k}$ we have the relation

$$
\begin{aligned}
\sum_{J \in 2^{I}}(-1)^{n-|J|} \mu\left(0+\sum_{i \in J} \Delta_{i}\right) & \\
= & \sum_{\Gamma \in \mathcal{R}_{0}(\Omega, k)}(-1)^{\operatorname{codim}(\Gamma)}\left(\sum_{J \in 2^{I}}(-1)^{n-|J|} \mu\left(0+\sum_{i \in J} \Gamma_{i}\right)\right),
\end{aligned}
$$

where $I=(1, \ldots, k)$ is the set of the first $k$ positive integers, and the sums over $J \in 2^{x}$ are taken over all subsets $J$ of the set $I$.

Proof. Starting with the measure $\mu$ on the space $L_{1}$ we construct a new measure $\bar{\mu}$ on the space $L_{1} \times L_{2}$ that contains a join $\Omega$ of the polytopes $\Delta_{1}, \ldots, \Delta_{k}$. The main theorem is obtained by applying Corollary 2 to this measure. We begin with the construction of an auxiliary measure $\mu_{J}$ on the space $L_{1} \times L_{2}$. Let $\Omega$ be the join of the polytopes $\Delta_{1}, \ldots, \Delta_{k} \subset L_{1}$ over a $(k-1)$-dimensional simplex, in a space $L_{2}$, with vertices $A_{1}, \ldots, A_{k}$. For any nonempty subset $J$ of the segment $I=(1, \ldots, k)$ of positive integers, denote by $L_{J}$ the affine space that is the preimage, under the projection $\pi: L_{1} \times L_{2} \rightarrow L_{2}$, of the point $A_{J} \in L_{2}$ given by the formula

$$
A_{J}=\frac{1}{|J|}\left(\sum_{i \in J} A_{i}\right),
$$

where $|J|$ is the number of elements of the set $J$. Denote by $\mathcal{B}_{J}^{0}$ the Boolean algebra (on the affine space $\left.L_{J}\right)$ that consists of the sets $\left(X, A_{J}\right)$ such that the set $|J| X$ (the homothetic dilation of the set $X$ with coefficient $|J|$ ) belongs to the Boolean algebra $\mathcal{B}_{\mu}$. For $\mu_{J}(X)$ we take $\mu(|J| X)$. This measure can be extended 
to the Boolean algebra $\mathcal{B}_{J}$ (on the space $L_{1} \times L_{2}$ ) that consists of the sets $Y$ such that $Y \cap L_{J} \in \mathcal{B}_{J}^{0}$ as follows: $\mu_{J}=\mu_{J}\left(Y \cap L_{J}\right)$. We can readily see that the value of the measure $\mu_{J}$ on the join of the polytopes $\Gamma_{1}, \ldots, \Gamma_{k}$ is $\mu\left(\sum_{i \in J} \Gamma_{i}\right)$.

We also introduce a measure $\mu_{\varnothing}$ for the empty subset $\varnothing \subset I$. Let $\mathcal{B}_{\varnothing}$ be the Boolean algebra generated by all convex polytopes $\Delta$ in the space $L_{1} \times L_{2}$. For the value of the measure $\mu_{\varnothing}$ on a polytope $\Delta$ we take $\mu(0)$, where $\mu(0)$ is the value of the measure $\mu$ on the singleton $\{0\} \subset L^{n}$. In other words, the measure $\mu_{\varnothing}$ is the measure $\mu(0) E$, where $E$ is the extension by additivity of the Euler characteristic to the Boolean algebra $\mathcal{B}_{\varnothing}$.

Finally we set

$$
\bar{\mu}=\sum_{J \in 2^{I}}(-1)^{n-|J|} \mu_{J},
$$

where the sum is taken over all subsets $J$ of the segment of positive integers (including the empty subset). The measure $\bar{\mu}$ is thus defined on the intersection of the Boolean algebras $\mathcal{B}_{J}$. The main theorem is proved by applying Corollary 2 of $\S 4$ to this measure.

Remark. In the proof of the theorem we used the existence of an extension of the Euler characteristic to the Boolean algebra $\mathcal{B}_{\varnothing}$. Instead of this, it suffices to apply assertion 2 of Corollary 2 from $\S 4$.

Corollary. For a correct partition $\mathcal{R}(\Omega)$ of a join of $n$ polytopes in $\mathbb{R}^{n}$, we have the identity

$$
V\left(\Delta_{1}, \ldots, \Delta_{n}\right)=\sum_{\Gamma \subset \mathcal{R}_{0}(\Omega, n)} V\left(\Gamma_{1}, \cdots, \Gamma_{n}\right) .
$$

The proof can be obtained by applying the main theorem to the measure $\mu$ that is equal to the ordinary Euclidean volume.

\section{§6. Polynomials on semigroups}

A function $f: G \rightarrow \mathcal{R}$ defined on a commutative semigroup $G$ with zero element is said to be a polynomial of degree $\leqslant k$ if, for any chosen elements $a_{1}, \ldots, a_{m}$ of the semigroup $G$, the function $f\left(\lambda_{1} a_{1}+\cdots+\lambda_{m} a_{m}\right)$ of the nonnegative integers $\lambda_{1}, \ldots, \lambda_{m}$ is a polynomial of degree at most $k$.

We shall need the classical Taylor formula in finite differences for functions on a lattice. To each element $a \in G$, two operators on the space of real functions $f$ on the semigroup $G$ are assigned: the shift operator $L_{a}$ defined by the formula $L_{a} f(x)=f(x+a), x \in G$, and the finite difference operator $D_{a}$ given by the formula $D_{a} f(x)=f(x+a)-f(x), x \in G$.

The shift operators and the finite difference operators are related as follows: $L_{a}=D_{a}+I$, where $I$ is the identity operator.

Taylor formula in finite differences. For any nonnegative integers $\lambda_{1}, \ldots, \lambda_{m}$ and for each function $f: G \rightarrow \mathcal{R}$, the following Taylor formula holds:

$f\left(\lambda_{1} a_{1}+\cdots+\lambda_{m} a_{m}\right)=\sum_{0 \leqslant k_{1} \leqslant \lambda_{1}, \ldots, 0 \leqslant k_{m} \leqslant \lambda_{m}}\left(\begin{array}{l}\lambda_{1} \\ k_{1}\end{array}\right) \cdots\left(\begin{array}{l}\lambda_{m} \\ k_{m}\end{array}\right)\left(D_{a_{1}}^{k_{1}} \cdots \cdots \circ D_{a_{m}}^{k_{m}} f\right)(0)$,

where $\left(\begin{array}{c}\lambda_{i} \\ k_{i}\end{array}\right)=\left(\lambda_{i}\left(\lambda_{i}-1\right) \cdots\left(\lambda_{i}-k_{i}+1\right)\right) / k !$ is the binomial coefficient, $\left(\begin{array}{c}\lambda_{i} \\ 0\end{array}\right)=1$, and $D_{a_{i}}^{0}=I$. 
Proof. We have

$$
\begin{aligned}
& f\left(\lambda_{1} a_{1}+\cdots+\lambda_{m} a_{m}\right) \\
& \quad=\left(L^{\lambda_{1}} \circ \cdots \circ L^{\lambda_{m}} a_{m} f\right)(0)\left(\left(D_{a_{1}}+I\right)^{\lambda_{1}} \circ \cdots \circ\left(D_{a_{m}}+I\right)^{\lambda_{m}}\right)(0) \\
& \quad=\sum\left(\begin{array}{l}
\lambda_{1} \\
k_{1}
\end{array}\right) \cdots\left(\begin{array}{l}
\lambda_{m} \\
k_{m}
\end{array}\right)\left(D_{a_{1}}^{k_{1}} \circ \cdots \circ D_{a_{m}}^{k_{m}} f\right)(0) .
\end{aligned}
$$

The Taylor formula in finite differences implies the following assertion.

Corollary. A function $f$ is a polynomial of degree $\leqslant k$ if, for an arbitrary choice of elements $a_{1}, \ldots, a_{k+1}$, the following relation holds:

$$
\left(D_{a_{1}} \circ \cdots \circ D_{a_{k+1}} f\right)(0)=0 .
$$

We say that $f$ is a polynomial function of degree $\leqslant k$ with respect to a subsemigroup $G_{0} \subseteq G$ containsing the zero element of $G, 0 \in G_{0}$, if, for any element $a \in G$, the restriction of the function $f_{a}(x)=f(a+x)$ to the semigroup $G_{0}$ defines a polynomial of degree $\leqslant k$ on $G_{0}$.

Proposition. Let $f$ be a polynomial function of degree $\leqslant k$ with respect to a subsemigroup $G_{0}$. Suppose that among the elements $a_{1}, \ldots, a_{m}$ of $G$, at least $k+1$ elements belong to the semigroup $G_{0}$. Then we have $D_{a_{1}} \circ \cdots \circ D_{a_{m}} f \equiv 0$.

Proof. Let us enumerate the elements $a_{1}, \ldots, a_{m}$ so that the first $k+1$ elements belong to the semigroup $G_{0}$. Then we have

$$
D_{a_{1}} \circ \cdots \circ D_{a_{m}} f=D_{a_{k+2}} \circ \cdots \circ D_{a_{m}}\left(D_{a_{1}} \circ \cdots \circ D_{a_{k+1}} f\right) \text {. }
$$

The function $D_{a_{1}} \circ \cdots \circ D_{a_{k+1}} f$ vanishes at any point $a \in G$ because the function $f_{a}(x)=f(x+a)$ is a polynomial on the semigroup $G_{0}$ of degree $\leqslant k$.

\section{§7. On POlynomial measures on the SPACE of POLYTopes}

We turn our attention to an application of the main theorem. Let $G_{0} \subset \mathbb{R}^{n}$ be an arbitrary semigroup with respect to the addition that contains the zero element. To the semigroup $G_{0}$ there corresponds a semigroup $G$ of polytopes (with respect to the addition given by the Minkowski sum) that consists of the polytopes all of whose vertices belong to the semigroup $G_{0}$.

Consider an arbitrary finitely additive measure $\mu$ on the Boolean algebra of sets generated by the polytopes from the semigroup $G$. We say that $\mu$ is a polynomial measure of degree $\leqslant k$ if, for any chosen polytope $\Delta \in G$, the function $f_{\Delta}(x)=$ $\mu(x+\Delta), x \in G_{0}$, is a polynomial of degree $\leqslant k$ on the semigroup $G_{0}$. This definition agrees with that in $\S 6$ in which a polynomial function with respect to a semigroup is defined.

Theorem. For any polynomial measure $\mu$ of degree $\leqslant k$ and for any chosen polynomials $\Delta_{1}, \ldots, \Delta_{m} \in G$, the function $\mu\left(\lambda_{1} \Delta_{1}+\cdots+\lambda_{m} \Delta_{m}\right)$ of the nonnegative integers $\lambda_{1}, \ldots, \lambda_{m}$ is a polynomial of degree $\leqslant n+k$.

Proof. To the measure $\mu$, a function $\mu: G \rightarrow \mathbb{R}$ on the semigroup $G$ is assigned so that $\mu$ maps each polytope $\Delta \in G$ to its measure $\mu(\Delta)$. According to the 
description of the polynomial functions on a semigroup, it suffices to show that for any collection of $n+k+1$ polytopes, say, $\Delta_{1}, \ldots, \Delta_{n+k+1} \in G$, the following relation holds:

$$
D_{\Delta_{1}} \circ \cdots \circ D_{\Delta_{n+k+1}} \mu(0)=0 .
$$

Consider a consistency of the tautological partitions of the polytopes $\Delta_{1}, \ldots$, $\Delta_{n+k+1}$ such that each of the consistent collections contains affinely independent faces of these polytopes (see the corollary in $\S 1$ ).

In any consistent collection of faces $\Gamma_{1}, \ldots, \Gamma_{n+k+1}$, at least $k+1$ faces are vertices because we have $\operatorname{dim} \Gamma_{1}+\cdots+\operatorname{dim} \Gamma_{n+k+1}=n$. In other words, at least $k+1$ polytopes from the collection $\Gamma_{1}, \ldots, \Gamma_{n+k+1}$ are elements of the semigroup $G_{0}$. By the proposition in $\S 6$, we have

$$
\left(D_{\Gamma_{1}} \circ \cdots \circ D_{\Gamma_{n+k+1}} \mu\right)(0)=0 .
$$

Furthermore, by the main theorem, we have the relation

$$
\left(D_{\Delta_{1}} \circ \cdots \circ D_{\Delta_{n+k+1}} \mu\right)(0)=\sum_{\Gamma \in \mathcal{R}_{0}(\Omega, n+k+1)}(-1)^{\operatorname{codim} \Gamma}\left(D_{\Gamma_{1}} \circ \cdots \circ D_{\Gamma_{n+k+1}} \mu\right)(0)=0 .
$$

Therefore, the measure $\mu$, regarded as a function on the semigroup $G$ of polytopes, is a polynomial of degree $\leqslant n+k$ (see the corollary in $\S 6$ ).

\section{REFERENCES}

1. D. Bernshtein, The number of roots of a system of equations, Funktsional. Anal. i Prilozhen. 9 (1975), no. 3, 1-4; English transl. in Functional Anal. Appl. 9 (1975), no. 3.

2. I. M. Gel'fand, M. M. Kapranov, and A. V. Zelevinsky, Discriminants, resultants, and multidimensional determinants, Birkhäuser, Boston, 1994.

3. O. Gel'fond and A. Khovanskiu,, Newton polytopes and Grothendieck residues, Dokl. Russian Akad. Nauk 3 (1996), no. 350, 298-300; English transl. in Russian Acad. Sci. Dokl. Math..

4. A. Khovanskiı̌, Algebra and mixed volumes, A Series of Geometry (Y. D. Burago and V. A. Zalgaller, eds.), vol. 285, Springer-Verlag, Berlin-New York, 1988, pp. 182-207.

5. A. Khovanskiı̌ and A. Pukhlikov, Finitely additive measures of virtual polytopes, Algebra i Analiz 4 (1992), no. 2, 161-185; English transl. in Leningrad Math. J. 4 (1993).

6. P. McMullen, Metrical and combinatorial properties of complex polytopes, Proc. Internat. Congr. Math. (Vancouver, 1974), Vol. 1, Canad. Math. Congr., Montreal, 1975, pp. 491-495.

7. O. Ya. Viro, Real algebraic varieties with prescribed topological properties, Thesis Doct. Math. (to appear in Transl. Math. Monographs, Amer. Math. Soc.), Leningr. Univ., Leningrad, 1983.

8. S_ Some integral calculus based on Euler characteristics, Topology and Geometry, Rokhlin Seminar, Lecture Notes in Math., vol. 1346, Springer-Verlag, Berlin, 1989, pp. 127-138.

Translated by A. I. SHTERN 